\title{
Note d'information et consentement des patients participant à des essais cliniques : vers des recommandations standardisées?
} \author{
$n^{\circ} 3$ de Giens XXIV* \\ 1 DRRC, PH-HP, Hôpital Saint Louis, Paris, France \\ 2 CNAFAL, Conseil National Associations Familiales Laïques, Paris, France \\ 3 Chemire Le Gaudin, France \\ 4 Sanofi-Aventis, Paris, France
}

Olivier Chassany ${ }^{1}$, Micheline Bernard-Harlaut ${ }^{2}$, Gilles Guy ${ }^{3}$, Nathalie Billon ${ }^{4}$ et les participants à la table ronde

Texte reçu le 15 octobre 2008 ; accepté le 20 novembre 2008

\section{Mots clés :}

consentement éclairé ; information; essais cliniques ; patients ; recherche biomédicale ; rédaction
Résumé - Les personnes qui participent à une recherche biomédicale doivent recevoir une information lisible et intelligible afin de pouvoir donner un consentement libre et éclairé. À ce jour, on note que les promoteurs ont tendance à apporter de nombreuses informations de différentes natures n'ayant pas toujours un rapport direct et réellement informatif pour les personnes qui se prêtent à cette recherche.

Ce constat a conduit à l'organisation d'une table ronde lors des rencontres de Pharmacologie Clinique et à une réflexion sur l'établissement d'une charte relative à la rédaction d'un document d'information des personnes participant à une Recherche Biomédicale ainsi que des bonnes pratiques de rédaction.

Les recommandations qui ont été faites, sont basées sur les travaux des différents groupes de travail tels que ceux de la Conférence Nationale des Comités de Protection des Personnes (CNCP), des représentants des usagers, des associations de patients, ceux des promoteurs industriels et institutionnels (CPI), et issus de la littérature.

La restitution de cette table ronde est structurée en 3 catégories et 14 points.

1) Le format : la conception; les règles de la rédaction; la mise en forme ; le sommaire et le lexique.

2) Le contenu : la page d'introduction ; la description de l'étude ; les risques et les bénéfices.

3) Les aspects réglementaires : les aspects Juridiques ; les mentions CNIL (Commission Nationale Informatique et Liberté); les aspects financiers; les conflits d'intérêts et les signatures multiples et variées.

Ce document doit contribuer à l'attractivité de la Recherche en France et il a été convenu de présenter la charte et ses documents après les rencontres de Pharmacologie Clinique et de les faire avaliser par l'ensemble des acteurs : DGS (Direction Générale de la Santé), Afssaps (Agence française de sécurité sanitaire des produits de santé), CNCP, CPI et Leem (Les entreprises du médicament).

Une fois, cette charte validée, elle pourra être mise à disposition des Promoteurs et des CPP (Comités de Protection des Personnes) afin de rendre l'information donnée aux patients uniforme et lisible.

\section{Introduction}

La nécessité d'écrire ces recommandations est devenue primordiale. Alors que les Personnes participant à la recherche biomédicale doivent recevoir une information lisible et intelligible et pouvoir donner un consentement libre et éclairé, les Promoteurs ont une nette tendance à apporter de nombreuses informations de

\footnotetext{
* Pour la liste des participants, voir en fin d'article.
}

différentes natures n'ayant pas toujours un rapport direct et réellement informatif pour les personnes qui se prêtent à la recherche.

Les CPP (Comités de Protection des Personnes) sont souvent embolisés par une rédaction confuse des documents soumis au dépens d'une réelle réflexion éthique et, doivent malgré ce, garantir une cohérence d'expertise et de décision d'un CPP à l'autre.

Les recommandations de Bonnes Pratiques de Rédaction du document d'information ont été regroupées en 8 points pour la 
restitution de table ronde. Ces recommandations sont basées sur les travaux des différents groupes de travail tels que ceux de la Conférence Nationale des Comités de Protection des Personnes $(\mathrm{CNCP}),{ }^{[1]}$ des représentants des usagers, des associations de patients, ceux des promoteurs industriels et institutionnels (CPI), et issus de la littérature. ${ }^{[2-9]}$

Les documents d'information et de consentement sont destinés aux personnes souhaitant participer à une Recherche Biomédicale et doivent permettre la compréhension des objectifs, des bénéfices et des risques, des contraintes et du déroulement du projet. Ces documents doivent être rédigés en français, avec des termes simples, sans ambiguité, sans longueur excessive, ils doivent contenir uniquement des informations utiles, sans répétition et doivent permettre de différencier le Soin de la Recherche Clinique. Le formulaire de recueil du consentement bien que signé, n'est pas un contrat juridique.

Le texte de ces recommandations doit correspondre à un réel engagement entre promoteurs et CPP. Ses principes sont guidés par la reconnaissance et le respect des personnes participant à la recherche, témoignant ainsi d'une démarche éthique forte de la part des promoteurs et des CPP au service de la recherche clinique. Ce document doit contribuer à l' attractivité de la Recherche en France et il a été convenu lors de la table ronde qu'il y avait une nécessité de présenter la charte et ses documents après les rencontres de Pharmacologie Clinique et de les faire avaliser par l'ensemble des acteurs : DGS (Direction Générale de la Santé), Afssaps (Agence française de sécurité sanitaire des produits de santé), CNCP, CPI et Leem (Les entreprises du médicament).

\section{Méthodes}

Les objectifs de la table ronde étaient d'établir une charte relative à la rédaction des documents d'information des personnes participant à la Recherche Biomédicale ainsi que des bonnes pratiques de rédaction. Ce premier travail ne concerne que le cas général, les questions particulières posées par les essais chez les enfants, les adolescents ou les personnes âgées n'ont pas été abordées.

\subsection{La conception}

La personne qui doit rédiger les documents d'information doit être une personne connaissant l'étude et la culture française, il s'agit donc du chef de projet de l'étude. Si le protocole et les annexes ont été écrits initialement en anglais, le chef de projet doit faire un contrôle de la traduction. Un comité de lecture doit vérifier cette rédaction et il nous semble nécessaire que l'investigateur principal relise le document d'information.
La rédaction de ce document doit reposer sur les 5 points-clé suivants :

\subsubsection{La distinction entre Recherche et Soin}

Dans une situation de soin, le médecin vise exclusivement l'intérêt du patient pris comme personne individuelle; il est uniquement guidé par l'intérêt direct du patient. Dans la situation de recherche, la visée est différente puisqu'il s'agit de produire une connaissance scientifique. On vise dans ce cas la collectivité dont la personne est un élément d'un groupe.

La reconnaissance de cette spécificité de la recherche biomédicale oblige à distinguer les situations de recherche des situations de soin.

Ainsi, on utilise les termes : médecin-investigateur, volontaire, produit à l'étude ou éligibilité pour décrire une situation de recherche biomédicale et on réserve les termes de médecin, patient, médicament ou traitement pour une situation de soin.

\subsubsection{Le consentement doit être éclairé}

La règle universellement admise aujourd'hui du consentement éclairé exprime l'obligation faite au médecin-investigateur d'informer la personne sollicitée de la nature et du contenu de l'objectif de recherche. Aussi l'information préalable doit porter d'abord sur le caractère spécifique de la situation de recherche.

Cette exigence est d'autant plus importante que dans le cas fréquent des recherches conduites dans un cadre de soin, le risque de confusion entre actes de soin et actes de recherche augmente. Les prises de sang ne disent pas d'elles-mêmes de quel type d'acte elles relèvent. Mettre d'abord la personne en situation de comprendre que c'est à une recherche biomédicale à laquelle on lui propose de participer est la première exigence de l'information.

Que la personne ait retenu une quantité de détails n'a pas d'intérêt si la distinction entre protocole de recherche et protocole de soin, entre produit étudié et médicament n'est pas établie.

\subsubsection{L'information doit être pertinente}

Sur cette base, la qualité de l'information dispensée est sa capacité à permettre à la personne de faire des choix (participer ou ne pas participer à la recherche).

L'intelligibilité d'une information permet de prendre des décisions.

L'idée souvent répétée qu'il faudrait «tout expliquer» est fausse. Une information pertinente est une information utile pour décider. Aussi les informations susceptibles de modifier la décision de la personne doivent être considérées comme primordiales 
(existence d'un risque, les conséquences de la participation sur la qualité de vie, les contraintes organisationnelles, etc.).

\subsubsection{L'information doit être destinée à la personne sollicitée pour participer à une recherche}

La reconnaissance de la spécificité de la situation de recherche biomédicale et l'évolution des dispositions législatives - qui attribue une responsabilité plus importante au promoteur conduisent à une valorisation de la procédure «écrite». Le document d'information ne peut plus être le simple accessoire d'une tradition orale, dont la responsabilité incomberait au médecininvestigateur. Ce document exprime l'engagement du promoteur à réaliser une recherche de qualité, respectueuse des personnes.

La personne perçoit, à travers la qualité des documents qu'on lui remet, le degré de considération qu'on lui porte. Elle doit être le premier destinataire de cette communication. Les marques de cette intention doivent être constantes.

\subsubsection{Les informations écrites et orales doivent être homogènes}

Les dispositions réglementaires précisent les conditions de délivrance de l'information : une information orale par le médecin-investigateur ou un médecin qui le représente et un document écrit, qui résume les informations orales. Les informations écrites et orales doivent être homogènes. L'articulation entre informations écrites et informations orales assure une même présentation des contenus. Les solutions de continuité pèsent sur le processus de décision de la personne, par les confusions qu'elles produisent.

\subsection{Les règles de la rédaction}

\section{a- Longueur des phrases}

Les phrases composées ne doivent pas comporter plus de 30 mots, la moyenne étant de 25 mots/phrase.

\section{$b$ - Construction des phrases}

On privilégie les phrases peu complexes. Les constructions par subordonnées successives sont à éviter. La présentation d'une seule information nouvelle par phrase permet la rédaction de phrases courtes parfaitement intelligibles.

\section{c-Vocabulaire spécialisé}

Le vocabulaire spécialisé qui mérite d'être précisé dépend de la culture médicale des personnes concernées par la spécialité médicale ou la pathologie. Certaines ont une bonne connaissance de leur pathologie, il est, en ce cas, maladroit de trop simplifier. La vulgarisation des termes et/ou des notions spécialisés, - quand elle a lieu, s'appuie sur les ressources " grand public " existantes.

\section{d-Style}

On choisit un style neutre. Les formes verbales sont actives plutôt que passives.

e-Respect de la terminologie

On veille à utiliser les termes et expressions d'un glossaire préalablement défini.

Cette recommandation vaut surtout pour les marqueurs de situation (situation de recherche biomédicale versus situation de soin). On porte une attention particulière à l'emploi des termes suivants : traitement, médicament, médecin-investigateur, recherche biomédicale.

\section{$f$ - La mise en page et l'orthotypographie}

On vérifie la bonne application de la règle des majuscules, de la ponctuation, etc. Les parenthèses seront à éviter et bien sûr les fautes d'orthographe et de grammaire sont à bannir. La taille de caractère utilisée devra être une police usuelle de taille 11/12, les paragraphes seront individualisés et aérés, et l'éventuel logo du promoteur devra rester discret.

\subsection{La mise en forme}

La mise en forme est importante afin de montrer à la personne qui souhaiterait participer à la Recherche l'intérêt et le respect que le promoteur a pour elle. La page de titre doit donner une valeur au document, le titre de l'étude et l'identité du promoteur doivent être précisés sur cette première page.

\subsection{Sommaire}

L'insertion d'un sommaire doit être réalisée en particulier s'il s'agit d'un document important ou long.

À titre d'exemple, nous proposons le sommaire suivant :

1. Le nom et le titre de l'étude

2. Le lexique

3. La page d'introduction

4. Le sommaire

5. Information générale sur le produit à l'étude

6. Information générale sur l'étude

7. Les visites

8. Les risques

9. Les bénéfices

10. Les alternatives médicales

11. L'interruption de l'étude

12. Les frais éventuels

13. Les droits et obligations

14. Le cadre réglementaire

15. Le tableau des visites 


\subsection{Lexique}

Il est également important d'ajouter un lexique pour l'information de la personne (aide à l'information orale). Ce lexique peut être composé :

- D'une partie de termes communs à l'ensemble des recherches biomédicales (consentement, recherche biomédicale, médecin-investigateur, promoteur, consultation, information, inclusion) avec leur définition.

- De termes spécifiques à la recherche biomédicale décrite dans le document. Chaque terme sera défini à partir de ressources « grand public» (dictionnaires ou sites internet).

- Le lexique qui figure en annexe I regroupe les termes recommandés pour la rédaction du document d'information, des exemples de termes à bannir ou à employer sont rassemblés.

\section{Résultats}

\section{1. La page d'introduction}

Elle se présentera sous forme d'une lettre qui s'adresse à la personne avec courtoisie et politesse et devra éviter «Cher Monsieur » ou «Cher Patient ». Notre proposition est simplement s'adresser à la personne : « Madame, Mademoiselle, Monsieur ».

L'objectif de l'étude devra clairement préciser en quoi la personne sollicitée est concernée et la mention d'un délai de réflexion sera indiquée bien que la précision de ce délai ne soit pas nécessaire. La liberté de choix de participer ou de refuser et l'engagement du médecin à prodiguer les meilleurs soins à la personne en cas de refus seront mentionnés.

\subsection{Description de l'étude}

La description de l'étude devra être rédigée en s'adressant à la personne ( «vous»), devra comporter la méthodologie utilisée et indiquer le déroulement de l'étude de façon claire avec l'utilisation de schémas et calendriers. Les modalités de prise en charge après l'étude ou en cas de sortie prématurée devront être précisées. Les mêmes règles de description doivent s'appliquer aux études ancillaires.

La durée de participation pour la personne ainsi que la durée totale de l'étude doivent être mentionnées. L'ajout d'une carte patient précisant certains points du protocole serait un plus.

Nous vous proposons de structurer cette partie comme suit, avec des sections et sous-sections qui peuvent être communes d'une recherche biomédicale à une autre, et d'autres qui devront être adaptées par la personne responsable de la rédaction de la notice.

\subsubsection{Information générale sur le produit à l'étude}

Conformément aux recommandations sur l'utilisation de terme simples et sans ambiguïté, on désigne par "produit à l'étude » ce qui est objet d'expérience, dans le cas des essais de médicaments. On proscrit l'expression «traitement à l'étude», sauf s'il s'agit de produits déjà évalués et employés.

\subsubsection{Type et statut}

On précise le type (molécule, dispositif, technique) et le statut au regard de la réglementation et des pratiques [AMM (Autorisation de Mise sur le Marché) ou pas, ATU (Autorisation Temporaire d'Utilisation) commercialisation, quel pays, quelle(s) indication(s)].

\subsubsection{Mécanisme d'action}

On présente le mode et le mécanisme d'action du produit ou du dispositif en termes clairs et simples. On réserve les explications techniques et scientifiques pour la partie orale de l'information, pour les personnes qui le souhaitent.

\subsubsection{Ce que l'on sait déjà}

Quel que soit le stade de développement, on mentionne les résultats des études précédentes. On peut, sous la forme d'un renvoi, livrer quelques éléments plus précis des études conduites. On donne, quand c'est le cas, les liens qui permettent la consultation complète des études citées.

\subsubsection{Information générale de l'étude}

Cette section concentre les informations utiles à la présentation de l'étude, indépendamment des aspects personnels d'une éventuelle participation à l'étude. On organise ces informations de la façon suivante :

\subsubsection{Contexte de la recherche}

On indique le contexte général de l'étude et la visée de «santé ». Pourquoi décide-t-on de mettre en œuvre une telle recherche (raisons et finalité(s)).

\subsubsection{Objectif(s)}

On précise l'objectif général de la recherche (ce que l'on cherche à savoir en priorité) ; on préfère insister sur l'objectif principal et ne pas multiplier les objectifs scientifiques secondaires. L'objectif de l'étude n'est pas confondu avec la finalité de l'essai (l'objectif peut être l'étude de la tolérance pour un essai dont la finalité est de tester une molécule pour un futur médicament). 


\subsubsection{Aspects méthodologiques}

On précise le nombre de centres ou de pays, le nombre de personnes concernées, la durée prévue et les dates, le schéma général du protocole (double aveugle placebo, tirage au sort). On se reportera au lexique pour la terminologie.

\subsubsection{Les visites}

La mise en œuvre de la recherche est décrite à la personne sous l'angle des visites, classées selon leur nature et leur ordre d'apparition : visite de sélection / visites d'inclusion et de réalisation de l'étude / visite de fin d'étude / visite(s) de suivi.

\subsubsection{La visite de sélection}

On liste à cet endroit en les classant par nature les actes prévus sur la personne par le protocole.

\subsubsection{La visite d'inclusion}

On précise le rôle de cette visite dans le déroulement de la recherche (confirmation de la participation par exemple) et les examens, tests ou bilans prévus.

\subsubsection{Les visites suivantes}

On décrit ici les aspects concrets et significatifs du point de vue de la personne de la mise en œuvre du protocole (quantité et type d'examens, contraintes, inconvénients, surveillance, conditions d'administration, etc.). Afin d'alléger cette section, on peut faire référence au tableau des visites sur lequel figure l'ensemble des actes prévus qu'il conviendrait d'insérer à la dernière page du document.

\subsubsection{La visite de fin d'étude}

On précise les examens, tests ou bilans prévus pour cette visite.

\subsubsection{La (es) visite(s) de suivi}

On indique leur nombre, leur périodicité, et le détail des examens, tests et bilans.

\subsubsection{Le calendrier des visites de l'étude}

Une représentation graphique de l'étude est souhaitable, adaptée aux caractéristiques du protocole (fréquence et nature des visites, contenus et détails des examens, présentations des différentes possibilités selon le schéma expérimental, etc.) Cette représentation graphique doit être claire et compréhensible sur le déroulement de l'étude du point de vue de la personne.

\subsubsection{L'interruption de l'étude}

La participation à une recherche biomédicale est un acte volontaire et non un engagement au sens formel ou contractuel. Cette participation peut être interrompue, pour diverses raisons. La section distingue plusieurs types de raisons :

- du fait du promoteur, de l'investigateur ou des autorités de santé,

- pour raisons médicales,

- du fait de la personne (désistement).

\subsection{Les Risques}

Nous vous proposons de regrouper les informations sur les effets indésirables, sur les examens et ceux liés à la grossesse.

La section « risques » se compose en 4 sous sections :

\subsubsection{Les effets et événements indésirables connus}

Ces informations devront être présentées clairement et honnêtement en reprenant ceux qui informent réellement la personne.

\section{Molécules en développement}

Il faudra préciser les événements indésirables (EI) déjà observés dans les essais précédents. Il ne sert à rien de présenter les résultats des études chez l'animal, in vitro ou in vivo, sauf pour les molécules en phase I ou II. Dans ce cas, les données présentées doivent réellement apporter une information compréhensible et utile à la personne.

\section{Dispositif}

Il faut préciser les risques d'éventuelles radiations sans les minimiser.

> Produits déjà commercialisés

La notice patient pourra être utilisée, le texte du résumé des caractéristiques du produit n'est pas adapté.

\section{Médicaments associés mais non fournis}

Les effets indésirables des médicaments associés mais non fournis (médicaments non expérimentaux), ne sont pas à présenter car prescrits dans le cadre de l'AMM, et faisant partie du traitement administré de toute façon dans le cadre du soin.

De manière générale, la présentation devra être réalisée en fonction de la gravité et de la fréquence (ainsi, on ne devra pas noyer l'aplasie au milieu des nausées et de la fatigue). 


\subsubsection{Les risques liés aux procédures}

Il s'agit autant des conséquences possibles des examens médicaux que de leurs conditions de réalisation, appréciées sous l'angle de la pénibilité pour les personnes (douleur notamment). Les informations sur les risques associés aux examens et procédures de la recherche doivent être en adéquation avec le risque réel de ces examens et avec la gravité de la pathologie. À titre d'exemple : ne pas exagérer les risques liés aux prises de sang, ne pas oublier les risques liés par exemple à une anesthésie, si un examen ajouté par la recherche nécessite une telle anesthésie, qui n'aurait pas été faite dans le cadre du soin habituel.

\subsubsection{Les risques liés aux situations particulières}

On indique les risques spécifiques encourus par les populations ou les situations particulières (allergies, grossesse, etc.). Il importe d'adapter les recommandations aux populations spécifiques de l'étude (patients jeunes, patients en phase terminale...) ainsi les informations liées à la grossesse ou allaitement doivent être en adéquation avec la population de l'étude, le risque potentiel des médicaments et la pathologie. De la même façon, les moyens contraceptifs proposés doivent être réalistes, adaptés à la personne et à l'étude.

\subsubsection{Les précautions à prendre}

On complète la section par l'exposé pratique des dispositions à prendre en cas de problème.

Ainsi, on appréciera la conduite à tenir en cas de survenue d'EI ou de signes d'appels (par ex. : aplasie/fièvre, saignement/anticoagulant...). Les signes directement perceptibles devront être mis en évidence.

\subsection{Les bénéfices}

On distingue trois types de bénéfices, qui sont décrits aussi précisément que possible. La présentation des bénéfices possibles (notamment le bénéfice personnel thérapeutique) ne doit pas être considérée comme une pression pour participer.

\subsubsection{Le bénéfice personnel thérapeutique}

Il doit être précisé en termes concrets (« réduction possible de votre risque de crise cardiaque » par exemple). En outre, les conditions de son observation doivent être explicites, ainsi le bénéfice personnel thérapeutique pourra être observé si le produit à l'étude se révèle efficace et si la personne a été incluse dans le groupe «produit à l'étude». On précise également le fait que ce bénéfice n'existe pas pour le groupe placebo.

\subsubsection{Le bénéfice personnel non thérapeutique}

Le fait de participer à une étude induit une prise en charge «améliorée » par rapport aux soins et traitements habituels. On précise cet avantage, en fonction des caractéristiques de l'étude (type d'examens, possibilités de diagnostic, etc.).

\subsubsection{Le bénéfice collectif}

Il existe toujours, par principe, un bénéfice collectif à la mise en œuvre d'une recherche biomédicale (rappel : la recherche biomédicale est définie par le législateur comme l'amélioration des connaissances biologiques et médicales).

\section{Les aspects juridiques}

Les aspects juridiques correspondent aux références légales relatives au Code de la Santé Publique; il est donc inutile d'énumérer les articles et leurs références. La phrase type « Nous vous proposons de participer à une Recherche Biomédicale, encadrée par la Loi destinée à vous protéger» est suffisante.

Il serait nécessaire qu'un groupe de travail formé de juristes soit créé pour vérifier si la phrase couramment retrouvée sur le «transfert des données Hors Europe » est suffisante pour assurer la protection des personnes. Notamment doit-on exiger des promoteurs d'ajouter la phrase suivante : le promoteur s'engage à assurer le niveau de sécurité équivalent à la Loi française ou européenne pour vos données envoyées à l'étranger.

\section{Les mentions CNIL}

Les mentions CNIL (Commission Nationale de l'Informatique et des Libertés) ne sont pas modifiables bien que ce texte ne réponde pas à nos recommandations d'intelligibilité et de lisibilité. Un groupe de travail CNCP, DGS, LEEM et CNIL serait nécessaire pour réfléchir sur une proposition d'un texte plus accessible.

\section{Les aspects financiers}

Les informations sur les Brevets et la propriété intellectuelle ne sont pas obligatoires car elles ne visent pas à protéger le patient.

\section{Assurance}

Concernant l'indemnisation, la mention de l'existence d'une assurance contractée par le promoteur est indispensable, par contre le numéro de la police n'est pas obligatoire. Des mesures ou des précautions visant à limiter la portée de l'assurance n'ont pas à figurer dans la notice d'information.

\section{Frais des personnes}

Les frais éventuels (les expliciter clairement) seront pris en charge en totalité par le promoteur. Pour le respect de la confidentialité des données par rapport au promoteur, le recours par un 
tiers de confiance est indispensable pour procéder à la gestion des remboursements.

\section{Indemnisation}

Quel que soit le contexte, une indemnité du fait des contraintes subies peut être versée à la personne. Dans ce cas, elle est à préciser dans le document d'information.

\section{Affiliation à un régime général de sécurité sociale}

L'affiliation à un régime de sécurité sociale ou bénéficiaire d'un tel régime (ayant droit) est à mettre dans la notice et le formulaire de recueil de consentement.

\section{Conflits d'intérêts}

La déclaration d'Helsinki recommande aux investigateurs de déclarer leurs conflits d'intérêt aux patients mais ceci peut se faire par oral. Il est à noter que l'explication à la personne des différentes sources de financement notamment lors de partenariats public-privé est plutôt une source de confusion. En toute rigueur, les conflits d'intérêts de l'investigateur sont ceux le liant au promoteur qui étudie une molécule ou un dispositif médical, mais aussi aux éventuels industriels concurrents.

En fonction de ces éléments, nous recommandons de ne pas imposer par écrit la déclaration de conflit d'intérêt de chaque investigateur, celui-ci peut le faire par oral.

\section{Signatures multiples et variées}

Le document devra être signé par le patient. Le patient pourra faire appel à un témoin uniquement quand il ne peut ou ne sait pas écrire. Pour les mineurs ou incapables majeurs seule la signature du Représentant légal sera nécessaire. Lors de situation d'urgence ou de coma, seule la personne habilitée par la Loi sera autorisée à signer.

La signature de l'Investigateur n'est pas requise par la Loi, mais elle concrétise un engagement de l'investigateur. La table ronde la recommande donc.

Les paraphes ne sont pas obligatoires, chaque page du document d'information doit comporter les références de l'étude (date, version). Notice et attestation de consentement pourraient constituer un même document non séparable.

\section{Conclusion}

Ces recommandations doivent devenir une réelle aide pour les promoteurs lors de rédaction de la notice d'information et pour les CPP lors de l'analyse des études qui leur sont soumises. Elles nécessiteront d'être avalisées par l'ensemble des partenaires Coordination des Promoteurs Institutionnels (CPI), LEEM, CNCP et par les différentes autorités (DGS, Afssaps).

\section{Participants}

Bernard Alberola (Wyeth Pharmaceuticals), Jean-François Bergmann (Médecine Interne A, Hôpital Lariboisière, Paris), Micheline Bernard-Harlaut (CNAFAL), Christian Cailliot (Amgen), Hélène Chappuy (Hôpital Necker Enfants Malades, Paris), Olivier Chassany (DRRC, AP-HP, Hôpital Saint Louis, Paris), Xavier Coron (Sanofi-Aventis), Jean-Luc Cracowski (CIC, Inserm, CHU, Grenoble), Hervé Decousus (Médecine Interne et Thérapeutique, CHU Hôpital de Bellevue, Saint-Étienne), Christophe Demonfaucon (AFTOC, Chateaufort), Catherine Fagard (Inserm, Université Bordeaux), Hugues Fischer (Association de patients, Paris), Odile Gelpi (Hospices civils, Lyons), Danielle Golinelli (Directions Générale de la Santé), Marie-Laurence Gourlay (Afssaps), Gilles Guy (Chemire le Gaudin), Yves Juillets (LEEM), Rémi Le Coent (GlaxoSmithKline), Christian Legrand (URAEUS, Fontainebleau), Monique Malchiodi (Laboratoires Roche), Anne-Laure Morin (Avocate, Paris), Véronique Nalet (Lilly France), Alain Olympie (La Maison des MICI, Paris), François Rivaille (laboratoires Servier), Claire Sibenaler (LLEM), Isabelle Thizon de Gaulle (Sanofi-Aventis).

\section{Références}

1. Groupe de travail sur le document d'information et le consentement éclairé de la Conférence Nationale des Comités de Protection des Personnes. Synthèse des travaux.

2. Paris A, Cracowski JL, Ravanel N, et al. Lisibilité de l'information écrite destinée aux sujets se prêtant à une recherche biomédicale. Presse Med 2005; 34: 13-8

3. Paris A, Cracowski JL, Maison P, et al. Impact of French "Comités de Protection des Personnes" on the readability of informed consent documents (ICD) in biomedical research: more information, but not better information. Fundam Clin Pharmacol 2005; 19: 395-9

4. Sharp SM. Consent documents for oncology trials: does anybody read these things? Am J Clin Oncol 2004; 27: 570-5

5. Wendler D. Can we ensure that all research subjects give valid consent? Arch Intern Med 2004; 164: 2201-4

6. Flory J, Emanuel E. Interventions to improve research participants' understanding in informed consent for research: a systematic review. JAMA 2004; 292: 1593-601

7. Bjørn E, Rossel P, Holm S. Can the written information to research subjects be improved? An empirical study. J Med Ethics 1999; 25: 263-7

8. Dresden GM, Levitt MA. Modifying a standard industry clinical trial consent form improves patient information retention as part of the informed consent process. Acad Emerg Med 2001; 8: 246-52

9. Paris A, Nogueira da Gama Chaves D, Cornu C, et al. Improvement of the comprehension of written information given to healthy volunteers in biomedical research: a single-blind randomized controlled study. Fundam Clin Pharmacol 2007; 21: 207-14

Correspondance et offprints : Nathalie Billon, Sanofi Aventis, 9 boulevard Romain Rolland, 75159 Paris Cedex 14, France.

E-mail : nathalie.billon@sanofi-aventis.com 


\section{ANNEXE 1}

\section{Lexique}

Nous vous présentons des propositions d'élaboration du lexique du formulaire d'information.

\section{1 / Alternatives médicales}

Ensemble des choix disponibles, en termes de traiteDÉFINITION ment et de soins, au moment de la sollicitation.

La décision de participer à une recherche doit être éclairée, c'est-à-dire informée par la connaissance de l'ensemble des possibilités existantes, y compris et surtout, sur le plan médical.

Exemples "Le médecin-investigateur vous informera des alter-
natives médicales disponibles. »

L'expression figure dans le texte de Loi. Article L.1122-1 : «Préalablement à la réalisation d'une recherche biomédicale sur une personne, l'investigateur, ou un médecin qui le représente, lui fait

Commentaires connaître notamment les éventuelles alternatives médicales. »

L'idée sous-jacente est qu'une personne ne doit pas accepter de participer à une recherche sans avoir eu une information complète sur l'ensemble des possibilités de prises en charge, au regard de sa pathologie. Le document d'information doit reprendre l'expression «Alternatives médicales ». On doit proscrire les autres expressions, plus ambiguës.

Voir la notion de «Traitement ». Une expression comme « Autres traitements disponibles » laisserait entendre que la recherche proposée est un traitement. Or la Loi Huriet repose sur la distinction entre « soin/ traitement » d'une part, et «recherche biomédicale » d'autre part.

Autres choix possibles / autres traitements / autres Termes proscrits choix thérapeutiques en dehors de la présente étude / traitements alternatifs / méthodes alternatives de traitement / alternative / autres traitements disponibles / autres choix thérapeutiques possibles / autres possibilités de traitement

\begin{tabular}{ll}
\hline 2 / ANONYMAT & \\
\hline DÉFINITION & Non divulgation de l'identité de la personne. \\
\hline Exemples & $\begin{array}{l}\text { "Les informations vous concernant seront traitées de } \\
\text { façon anonyme... } \\
\text { "L'anonymat des données sera assuré par... » }\end{array}$ \\
\hline
\end{tabular}

Commentaires

- Pas de difficulté sur la notion. La traduction concrète de cette notion est l'application des règles du secret professionnel médical à la recherche biomédicale. Elle signifie concrètement que l'identité de la personne ne sera pas divulguée.

Termes Proscrits Préservé/ secret

\begin{tabular}{ll}
\hline 3 / AssuranCE / Contrat d'assurance \\
\hline Exemples & $\begin{array}{l}\text { «Conformément aux dispositions de ce Code, le pro- } \\
\text { moteur a souscrit un contrat d'assurance. » }\end{array}$ \\
\hline Commentaires & $\begin{array}{l}\text { Notion sans difficulté. Le public est habitué au } \\
\text { principe de l'assurance. }\end{array}$
\end{tabular}

Termes proscrits Assurance souscrite

\begin{tabular}{|c|c|}
\hline \multicolumn{2}{|c|}{4 / AtTESTATION dE CONSENTEMENT } \\
\hline DÉFINITION & $\begin{array}{l}\text { Document signé par la personne sollicitée, après } \\
\text { qu'elle a accepté de participer à la recherche qu'on } \\
\text { lui propose. }\end{array}$ \\
\hline Exemples & $\begin{array}{l}\text { "Vous gardez, même après avoir signé l'attestation } \\
\text { de consentement, le droit d'interrompre à tout mo- } \\
\text { ment votre participation à l'étude, sans avoir à vous } \\
\text { justifier. » }\end{array}$ \\
\hline Commentaires & $\begin{array}{l}\text { - L'expression «formulaire d'attestation de } \\
\text { consentement» désigne le document (papier } \\
\text { ou électronique) qui permet d'attester son } \\
\text { consentement à la participation à une recherche } \\
\text { biomédicale, en tant que document-type. } \\
\text { - On proscrira les expressions telles que «consen- } \\
\text { tement écrit». Le consentement est l'acte de } \\
\text { consentir; la signature est ce par quoi une per- } \\
\text { sonne atteste (matérialise) qu'elle a consenti. }\end{array}$ \\
\hline
\end{tabular}

Formulaire de consentement / formulaire de notice

Termes proscrits d'information / exemplaire de ce consentement / formulaire d'information / formulaire d'information et de consentement / feuille de consentement / attestation / formulaire de consentement éclairé/ consentement écrit / consentement de participation / lettre de consentement / document de libre consentement

\section{5 / AUTORISATION}

- Le terme recouvre deux notions :

- L'autorisation de conduire la recherche. Il s'agit de l'autorisation administrative, qui ne concerne pas directement la personne. La référence aux autorités de santé, à la CNIL et au comité de protection des personnes suffit pour situer la recherche dans un cadre autorisé

- L'autorisation donnée par un tiers (parent, personne de confiance ou autre) de réaliser une recherche biomédicale sur une personne incapable juridiquement ou psychologiquement de consentir («consentement par tiers »).

Commentaires

On évoquera l'autorisation de réaliser la recherche en mentionnant l'avis favorable du comité des protections des personnes, qui a valeur d'autorisation selon les dispositions législatives en vigueur.

Termes proscrits Permission/accord 


\begin{tabular}{ll}
\hline 6/ AUtORITÉ(s) DE SANTÉ \\
\hline DÉFINITION & $\begin{array}{l}\text { Organisme(s) ou institution(s) qui dispose(nt) d'un } \\
\text { pouvoir de réglementation, de contrôle ou de sur- } \\
\text { veillance en matière de recherche biomédicale. }\end{array}$ \\
\hline$\underline{\text { Exemples }}$ & $\begin{array}{l}\text { "Les données peuvent être transmises aux autorités } \\
\text { de santé, dans des conditions garantissant leur confi- } \\
\text { dentialité et leur anonymat. » }\end{array}$ \\
\hline Commentaires & $\begin{array}{l}\text { - On utilise exclusivement l'expression « autorité } \\
\text { de santé », qui désigne, selon les cas, l'Afssaps } \\
\text { et/ou la DGS. }\end{array}$
\end{tabular}

Sanitaires / autorités nationales / Afssaps / autorités

Termes proscrits de santé nationales et internationales / autorités de santé habilitées / hautes autorités / autorités de santé françaises ou étrangères habilitées / autorités de santé concernées / autorités sanitaires habilitées / autorités de tutelle locales ou étrangères / tiers habilités / autorités réglementaires étrangères / autorités sanitaires françaises ou internationales /autorités de tutelle / organisme de réglementation

\begin{tabular}{ll}
\hline 7 / AVIS DU COMITÉ DE PROTECTION DES PERSONNES / avis \\
\hline DÉFINITION & $\begin{array}{l}\text { Résultat de l'évaluation conduite par le comité de } \\
\text { protection des personnes (CPP) saisi. L'avis est fa- } \\
\text { vorable ou défavorable. }\end{array}$ \\
\hline$\underline{\text { Exemples }}$ & $\begin{array}{l}\text { "L'étude XXX a reçu un avis favorable... " } \\
\text { "L'avis rendu par le Comité de protection des per- } \\
\text { sonnes doit être... }\end{array}$ \\
\hline
\end{tabular}

Commentaires - Mention obligatoire dans le document d'information, qui renvoie à une procédure peu connue du grand public qui ignore pour l'essentiel le rôle et la mission des CPP.

\begin{tabular}{ll}
\hline Termes proscrits & Avis éclairé \\
\hline $\mathbf{8 / \text { BÉNÉFICE }}$ & \\
\hline DÉFINITION & $\begin{array}{l}\text { Tout avantage produit ou obtenu du fait de la réalisa- } \\
\text { tion de la recherche. }\end{array}$ \\
\hline Exemples & $\begin{array}{l}\text { "Un bénéfice personnel thérapeutique est possible si } \\
\text { vous faîtes partie du groupe... } \\
\text { "Votre participation apporte un bénéfice à la collec- } \\
\text { tivité en fournissant... " }\end{array}$ \\
\hline
\end{tabular}

\section{Commentaires}

- Le terme «bénéfice » recouvre plusieurs notions ou sous notions :

- Le bénéfice personnel thérapeutique ;

- Le bénéfice personnel non thérapeutique;

- Le bénéfice collectif de la réalisation de la recherche;

○ La balance ou le rapport «bénéfices/risque».
- La notion de «bénéfice thérapeutique » fondait l'ancienne distinction entre "recherches thérapeutiques » et « recherches non thérapeutiques. » Elle désigne aujourd'hui un bénéfice personnel conditionnel (possible mais non garanti).

- La notion de «bénéfice individuel, » abandonnée par le législateur (texte du 9 août 2004) est ambiguë et son usage reste délicat.

- La notion de bénéfice collectif renvoie au fait que la recherche biomédicale a comme but et comme justification l'augmentation des connaissances biologiques et médicales. Ce bénéfice est pris en compte pour l'évaluation de la balance bénéfices/risques.

- Chaque type de bénéfice doit être décrit précisément (contenus et conditions de réalisation).

Bénéfice potentiel / retirer un bénéfice / bénéfice possible / bénéfice personnel supplémentaire / bénéfice

Termes proscrits personnel direct / bénéfice individuel direct / bénéfice thérapeutique / bénéfice attendu / effet favorable / bénéfice clinique / bénéfice direct / bénéfice prévisible / votre intérêt

\section{9 / CARTE DE PARTICIPATION}

\begin{tabular}{ll}
\hline DÉFINITION & $\begin{array}{l}\text { Document remis à la personne qui a accepté de par- } \\
\text { ticiper à une recherche biomédicale, qui atteste de sa } \\
\text { participation. }\end{array}$ \\
\hline Exemples & $\begin{array}{l}\text { "Ayez toujours votre carte de participation sur } \\
\text { vous. » } \\
\text { "Lors des consultations, présentez votre carte de } \\
\text { participation à votre médecin. » }\end{array}$ \\
\hline
\end{tabular}

Commentaires

- Notion qui ne pose pas de difficulté. L'intitulé exact précise «carte de participation à une recherche biomédicale », que l'on doit citer intégralement quand le contexte est ambigu.

- L'expression «carte/patient» en vigueur doit être proscrite et réservée à la logique et au protocole de soin.

$\overline{\text { Termes proscrits Carte patient / carte d'information / carte indiquant }}$ votre participation à l'étude / carte

\section{0 / COMItÉ DE PROTECTION DES PERSONNES (CPP)}

\begin{tabular}{ll}
\hline DÉFINITION & $\begin{array}{l}\text { Structure administrative chargée de l'évaluation des } \\
\text { protocoles de recherche biomédicale, préalablement } \\
\text { à toute mise en œuvre. }\end{array}$ \\
\hline Exemples & $\begin{array}{l}\text { "Cette étude a été soumise au Comité de protection } \\
\text { des personnes de la Pitié Salpêtrière... " }\end{array}$ \\
\hline
\end{tabular}


Commentaires

- La mention de l'avis est obligatoire. Le public connaît mal ou peu le dispositif (composition, mission).

- On proscrit l'emploi des seules initiales CPP dans le document d'information et l'attestation de consentement.

Comité indépendant / comité d'éthique / CCPPRB/ CPP / comité d'experts indépendants / comité de surveillance indépendant /commission de contrôle / organisme indépendant / comité d'éthique indépendant / comité institutionnel de révision

\section{1 / Confidentialtí́}

DÉFINITION Notion administrative : «maintien, - au sens de préservation ou de non divulgation-, des informations relatives aux personnes. »

\section{Exemples}

«Les informations recueillies seront traitées de façon confidentielle."

«La confidentialité des données est assurée par... »

- La notion de confidentialité est associée au secret professionnel et aux dispositions de la Loi «Informatique et Liberté. » Les règles de la confidentialité des données et les dispositions et modalités d'accès aux données sont précisées à l'article L.1121-3.

- On doit distinguer, dans l'information aux personnes, « confidentialité » et « anonymat »...

Termes proscrits Données à caractère personnel / préservée / secret professionnel / sans violation de la confidentialité

\section{2 / Consentement / Consentir / AcCepter / ACCord}

DÉFINITION Accord donné par la personne sollicitée pour participer à l'étude qu'on lui propose.

Exemples «Votre consentement ne décharge pas les organisa-
teurs de la recherche...»

«Si vous acceptez de participer... "

- Notion centrale de la mise en œuvre des recherches biomédicales. Les conditions de validité d'un consentement sont les suivantes : information du sujet (consentement éclairé), absence de pression ou liberté de choix (consentement libre), attestation (consentement exprès).

- L'expression «consentement écrit» est impropre et ne doit pas être utilisée.

- Il convient de distinguer entre le consentement donné par la personne même et les autorisations de mener le recherche, quand il s'agit d'un tiers (on ne peut consentir pour un tiers).

Termes proscrits Éclairé / accord de participation / - exprès / - libre, éclairé et exprès

\begin{tabular}{ll}
\hline $\mathbf{1 3}$ / DÉCISION & \\
\hline DÉFINITION & $\begin{array}{l}\text { Acte par lequel la personne sollicitée marque son ac- } \\
\text { cord ou son refus de participer à la recherche biomé- } \\
\text { dicale qu'on lui présente. }\end{array}$ \\
\hline \begin{tabular}{ll} 
Exemples & "Sanofi-Aventis a conçu ce document pour vous ai- \\
& $\begin{array}{l}\text { der à prendre votre décision... } \\
\text { ne doit jamais étre précipitée... }\end{array}$ \\
\hline Commentaires de participer ou de ne pas participer
\end{tabular} \\
\hline Termes proscrits & Avis éclairé \\
\hline
\end{tabular}

\begin{tabular}{|c|c|}
\hline \multicolumn{2}{|c|}{14 / DÉLAI DE RÉFLEXION } \\
\hline DÉFINITION & $\begin{array}{l}\text { Période ou temps qui sépare la sollicitation et la pré- } \\
\text { sentation de la recherche de la décision de participer } \\
\text { ou de ne pas participer de la personne sollicitée. }\end{array}$ \\
\hline Exemples & $\begin{array}{l}\text { "Vous disposerez d'un délai de réflexion avant de } \\
\text { prendre votre décision. » }\end{array}$ \\
\hline Commentaires & $\begin{array}{l}\text { - Notion sans difficulté. Dépend de l'étude. Il doit } \\
\text { toujours être précisé dans le document d'infor- } \\
\text { mation. }\end{array}$ \\
\hline
\end{tabular}

Termes proscrits Réflexion

\section{5 / DoCUMENT D'INFORMATION / DOCUMENT / BRoChURE /}

\section{LIVRET}

DÉFINITION Document remis à la personne sollicitée qui résume l'ensemble des informations relatives à l'étude proposée.

Exemples "Sanofi-Aventis France a conçu ce document d'information pour vous aider à prendre votre décision en toute connaissance de cause. »

- Le document d'information est un document spécifique, même si la règle lie, jusqu'à en faire une contrainte quasi-réglementaire, ce document à « l'attestation de consentement. »

Commentaires

- Le terme «notice» très usité doit être proscrit, pour éviter, notamment, la confusion avec les notices des médicaments.

- Le document d'information est obligatoire. La Loi (texte du 9 août 2004) précise que les comités de protection des personnes rendent leur avis sur les conditions de validité de la recherche, notamment au regard de... «l'adéquation, l'exhaustivité et l'intelligibilité des informations écrites à fournir ainsi que la procédure à suivre pour obtenir le consentement éclairé... »

Termes proscrits Feuillet d'information / feuille d'information / notice d'information / formulaire d'information / note d'information / formulaire de notice d'information et consentement éclairé / note écrite / notice d'information et consentement éclairé / lettre d'information / livret d'information et d'aide à la décision / résumé écrit / documentation 


\begin{tabular}{ll}
\hline 16 / DoNNÉES DE L'ÉTUDE \\
\hline DÉFINITION & $\begin{array}{l}\text { Informations collectées au cours de la réalisation de } \\
\text { l'étude ou produites par l'étude. }\end{array}$ \\
\hline Exemples & $\begin{array}{l}\text { Les données de l'étude pourront être transmises aux } \\
\text { autorités de santé... » }\end{array}$ \\
\hline Commentaires & $\begin{array}{l}\text { Notion sans difficulté. La personne qui participe } \\
\text { a peu à voir avec les données de l'étude en tant } \\
\text { que telles. } \\
\text { - Le terme est peu ou mal fixé et on trouve de } \\
\text { nombreuses variantes : «la nature des données à } \\
\text { caractère personnel recueillies», "j'accepte que } \\
\text { mes données ». } \\
\text { - On gardera, pour la communication, le caractère } \\
\text { neutre : «les données de l'étude ». }\end{array}$ \\
\hline Termes proscrits & $\begin{array}{l}\text { Données recueillies / données de base / données } \\
\text { source }\end{array}$ \\
\hline
\end{tabular}

17 / Double AVeugle / aveugle

DÉFINITION Procédure au cours de laquelle ni les investigateurs (chercheurs) ni les sujets testés ne savent qui reçoit ou non le produit à l'étude.

Exemples "Les volontaires sont répartis en deux groupes. Les membres du premier groupe prennent... Dans l'autre groupe, chacun reçoit un comprimé... Personne ne connaît la composition des groupes, ni vous, ni le médecin-investigateur.

Cet essai est dit en "double aveugle». Double aveugle signifie...»

- L'expression «double aveugle» relève du vo-

Commentaires cabulaire spécialisé et doit être expliquée. Voir l'exemple.

- Lorsque l'essai est en double aveugle, la définition doit figurer dans le lexique.

Termes proscrits Double insu / mise en insu

\begin{tabular}{ll}
\hline $\mathbf{1 8}$ / EFFET(S) INDÉSIRABLE(S) \\
\hline DÉFINITION & $\begin{array}{l}\text { Toute manifestation nocive et non désirée survenant } \\
\text { chez une personne qui participe à une recherche bio- } \\
\text { médicale. }\end{array}$ \\
\hline$\underline{\text { Exemples }}$ & $\begin{array}{l}\text { "En cas d'effet indésirable, prévenez immédiatement } \\
\text { votre médecin.... }\end{array}$ \\
\hline
\end{tabular}

- On distingue, selon le degré d'imputabilité :

- L'effet indésirable, grave ou non grave (relation au produit) :

Commentaires

- L'événement indésirable, attendu ou non attendu, grave ou non grave (quelle que soit la relation),

- Le modèle pour le grand public est principalement la notice d'information des médicaments, qui emploie l'expression « effet indésirable».

- On note que le glossaire de l'Afssaps retient le terme «effet indésirable » en se fondant sur l'article R.1123-39 du Code de santé publique.

- Le choix de l'expression ( «effet» ou "événement») se fera en tenant compte de la notion d'imputabilité.
Effet plus ou moins gênant / effet non souhaité / évé-

Termes proscrits nements anormaux / événement inattendu / symptôme anormal / symptôme imprévu / symptôme inhabituel / réaction indésirable / effets non connus / effets imprévisibles / désagréments / effet grave / toxicités inattendues / toxicités non listées / toxicité potentielle / effet inattendu / /problème grave / complication

\begin{tabular}{ll}
\hline 19/ ÉLIGIBILITÉ & / éligible \\
\hline DÉFINITION & $\begin{array}{l}\text { Qualité de la personne qui remplit les conditions re- } \\
\text { quises pour participer à une recherche biomédicale. }\end{array}$ \\
\hline \begin{tabular}{l} 
Exemples \\
\hline
\end{tabular} & $\begin{array}{l}\text { Lors de la visite de sélection, le médecin- } \\
\text { investigateur vérifiera que vous êtes éligible... » }\end{array}$ \\
\hline
\end{tabular}

- La procédure d'inclusion d'une personne sollicitée est mal connue du grand public. La difficulté est de parvenir à une présentation cohérente avec le vécu des personnes.

- Toute personne sollicitée n'est pas nécessairement incluse. Les différentes étapes de l'inclusion doivent être parfaitement décrites. On recommande de distinguer entre visite de sélection et visite d'inclusion quand cela est possible.

Apte / critères d'éligibilité / présélection / recrutement / sélection / période de sélection / visite de sélection / vérifier les critères de sélection / critères

Termes proscrits d'inclusion et de non inclusion / période d'inclusion / processus de sélection / patient incluable /

\section{0 / Fin DE L'ÉTUdE}

DÉFINITION Moment auquel s'arrête la recherche biomédicale ou la participation d'une personne à cette étude.

Exemples "À la fin de l'essai, le médecin-investigateur vous
informera des mesures à prendre...

- Notion sans difficulté apparente. Pourtant, la durée de l'essai n'est pas toujours identique à la durée de la participation.

Commentaires

- La notion de «fin d'essai » varie selon les acteurs, participants, investigateurs ou promoteurs : fin de l'administration des produits, fin des visites ou des consultations, fin de l'analyse des données, ou publication des résultats.

- On note qu'une fin de participation n'est pas la fin de l'essai ; il convient de distinguer fin de l'essai et interruption de la participation.

- On propose de réserver l'expression «fin de l'essai » pour la clôture de la recherche et « fin de la participation » ou «interruption de la participation » pour la personne qui participe.

Termes proscrits Clôture 


\section{1 / FraIS /Coûts}

DÉFINITION Ensemble des dépenses occasionnées par la réalisation de l'étude.

\begin{tabular}{ll}
\hline Exemples & "Les coûts de l'étude sont pris en charge en totalité \\
& par Sanofi-Aventis... \\
& "Les frais éventuels occasionnés par votre partici- \\
& pation...
\end{tabular}

- Notion qui ne pose pas de difficulté. Le terme « coût» s'applique à l'ensemble de la recherche

Commentaires (sommes dépensées pour la réalisation de la recherche); celui de «frais » vise les dépenses locales ou individuelles. La personne qui participe à la recherche est sensible aux frais (et à leurs remboursements), moins aux coûts.

- On propose de maintenir dans la section «Coûts » la distinction entre les deux termes (Les coûts de l'étude sont pris en charge... / Les frais éventuels occasionnés par votre participation...).

Termes proscrits Non documenté

\begin{tabular}{ll}
\hline $\mathbf{2 2} /$ INCLUSION / visite d'inclusion \\
\hline DÉFINITION & $\begin{array}{l}\text { Confirmation de la participation d'une personne à } \\
\text { une recherche biomédicale. }\end{array}$ \\
\hline \begin{tabular}{l} 
Exemples \\
\hline $\begin{array}{l}\text { La visite d'inclusion a lieu si vous décidez de par- } \\
\text { ticiper à l'étude... » }\end{array}$
\end{tabular} \\
\hline
\end{tabular}

- La procédure d'inclusion d'une personne sollicitée est mal connue du grand public. La difficulté est de parvenir à une présentation cohérente avec le vécu des personnes.

- Toute personne sollicitée n'est pas nécessairement incluse. Les différentes étapes de l'inclusion doivent être parfaitement décrites. On recommande de distinguer entre visite de sélection et visite d'inclusion quand cela est possible.

Termes proscrits Apte / critères d'éligibilité / éligibilité / préselection / recrutement / sélection / période de sélection / visite de sélection / vérifier les critères de sélection / critères d'inclusion et de non inclusion / période d'inclusion / processus de sélection / patient incluable

\section{3 / INDEMNITÉ FINANCIÈRE / Indemnité de compensation}

\begin{tabular}{ll}
\hline DÉFINITION & $\begin{array}{l}\text { Somme versée sous certaines conditions à une per- } \\
\text { sonne qui participe à une recherche biomédicale, au } \\
\text { titre des contraintes subies. }\end{array}$ \\
\hline$\underline{\text { Exemples }}$ & $\begin{array}{l}\text { "La participation à l'étude ne donne lieu par ailleurs } \\
\text { à aucune indemnisation financière. » }\end{array}$ \\
\hline
\end{tabular}

- Le terme « indemnisation » est compris généralement comme « dédommagement ». Il convient de maintenir des distinctions entre les notions de « rémunération », « d'indemnisation » et de «remboursement des frais engagés ou subis».

- La rémunération est exclue par le législateur qui ne retient que le principe d'une indemnité de compensation des contraintes subies. Quand elle n'est pas prévue, seul subsiste le remboursement des frais exposés.

Termes proscrits Compensation financière / rémunération / indemnisation / contrepartie financière

\section{4 / INFORMATION}

DÉFINITION Ensemble des moyens et des procédures qui visent à présenter et à expliquer à la personne sollicitée la proposition qu'on lui fait de participer à une recherche biomédicale.

Exemples «Ce document a pour but de vous fournir les infor-
mations nécessaires à votre décision. "

"L'information doit être objective, claire, loyale et permanente.»

Commentaires

- Notion centrale dans le dispositif de protection des personnes, mais peu problématique en soi. Le public est familiarisé avec l'idée et les pratiques de l'information.

\section{Termes proscrits Explications}

\begin{tabular}{ll}
\hline $\mathbf{2 5} /$ INTERRUPTION \\
\hline DÉFINITION & $\begin{array}{l}\text { Cessation avant la date prévue de la participation ou } \\
\text { de la réalisation de la recherche biomédicale. }\end{array}$ \\
\hline$\underline{\text { Exemples }}$ & $\begin{array}{l}\text { "L'étude peut être interrompue pour tous les partici- } \\
\text { pants... } \\
\text { "Vous pouvez, à tout moment, décider d'interrompre } \\
\text { votre participation... . }\end{array}$ \\
\hline
\end{tabular}

- L'interruption peut être celle de l'étude ou de la participation. Il convient de distinguer les différents cas de figure; le plus souvent, on vise l'arrêt de la participation de la personne. On précise selon le cas :

○ L'interruption volontaire (désistement, retrait);

- L'interruption pour des raisons médicales (décision de l'investigateur);

- L'interruption pour tous les participants (arrêt de l'étude), par décision du promoteur ou des autorités de santé.

Termes proscrits Désistement / arrêt / retrait 


\begin{tabular}{ll}
\hline 26/ LIBERTÉ / libre de \\
\hline DÉFINITION & $\begin{array}{l}\text { Possibilité pour la personne sollicitée de pouvoir se } \\
\text { décider sans contrainte et sans pression d'aucune } \\
\text { sorte. }\end{array}$ \\
\hline $\begin{array}{l}\text { Exemples } \\
\text { "Vous êtes libre d'accepter ou de refuser de partici- } \\
\text { per à cette recherche. » }\end{array}$ \\
\hline
\end{tabular}

- Notion essentielle du point de vue réglementaire et éthique, mais qui pose peu de problème en soi. Les problèmes rencontrés visent :

Commentaires

- Les cas particuliers (incapables juridiques ou psychologiques);

- Les incitations (formulations ambiguës qui limitent la liberté de choix).

Le respect de la liberté de choix s'exprime notamment par la mise à même niveau de l'acceptation et du refus de participer à la recherche biomédicale.

Termes proscrits Autonomie

\begin{tabular}{ll}
\hline 27 / LIEU DE RECHERCHE \\
\hline DÉFINITION & Endroit où se déroule la recherche. \\
\hline Exemples & $\begin{array}{l}\text { "La recherche se déroulera au Centre d'investigation } \\
\text { clinique de l'hôpital... » }\end{array}$ \\
\hline
\end{tabular}

Commentaires

- La notion est administrative, liée à la question de l'autorisation des «lieux de recherche ». Elle n'a de sens pour la personne que s'il y a confusion ou incertitude sur l'endroit où doit se rendre le sujet pour l'essai.

Termes proscrits Centre investigateur / site investigateur / milieu hospitalier / centre / centre d'investigation / centre de recherche

\section{8 / MÉdECIN (votre)}

DÉFINITION Personne qui dispense de façon habituelle les soins et les traitements.

Exemples «Votre médecin continuera de vous prescrire..."

- La notion visée est ici la personne en charge des soins du sujet sollicité. C'est la personne que l'on va voir pour se soigner.

Commentaires

- La distinction entre le médecin et l'investigateur est primordiale, même si le médecin qui fait la proposition peut devenir, avec l'inclusion du sujet dans l'essai, le médecin-investigateur de l'étude.

- Ici, la terminologie suit les fonctions plus que les personnes.

Termes proscrits Médecin traitant / médecin généraliste / votre médecin de famille

\section{9 / MÉDECIN-INVESTIGATEuR / Investigateur}

DÉFINITION Personne chargée de la mise en œuvre de la recherche biomédicale

$\begin{array}{ll}\text { Exemples } & \text { "Le médecin-investigateur répond à toutes vos ques- } \\ & \text { tions. » } \\ & \text { "Personne ne connaît la composition des groupes, ni } \\ & \text { vous, ni le médecin-investigateur. » }\end{array}$

- Le médecin-investigateur est la personne en charge de la mise en œuvre de la recherche. Le terme offre l'avantage à la fois d'être un marqueur de situation clair (médecin = soin; médecin-investigateur $=$ recherche) et d'indiquer le cadre médical (attention portée à la santé des personnes) dans lequel se déroule une recherche biomédicale.

- On propose de réserver les expressions aux domaines qu'ils désignent de cette façon :

○ Le terme «médecin» utilisé seul ne doit jamais désigner l'investigateur de l'étude. Il est réservé à l'activité et aux procédures du soin (le médecin propose l'étude, le médecininvestigateur la conduit);

○ Les termes « investigateur-principal », « investigateur coordonnateur » et « investigateur secondaire » renvoient aux dispositions administratives et organisationnelles; elles intéressent peu les sujets. L'information pratique consiste à indiquer clairement la personne responsable à contacter sur les lieux de l'essai.

Médecin en charge de l'étude (médecin de cette étude) / votre médecin investigateur / médecin chargé de l'étude / médecin de l'étude / médecin conduisant la recherche biomédicale / votre médecin de recherche / votre médecin de l'étude / médecin responsable de cette étude / médecin coordinateur / chercheur / personnel de l'étude / personne désignée du service / personne autorisée

\section{0 / MÉdicament}

Article L5111-1 (CSP) : «On entend par médicament DÉFINITION toute substance ou composition présentée comme possédant des propriétés curatives ou préventives à l'égard des maladies humaines ou animales, ainsi que tout produit pouvant être administré à l'homme ou à l'animal en vue d'établir un diagnostic médical ou de restaurer, corriger ou modifier leurs fonctions organiques. »

Exemples
vous avez besoin... m


- Le terme «médicament » renvoie pour le public à un produit déjà évalué, qui possède des qualités Commentaires thérapeutiques (qui soigne). Le Petit Robert indique en effet : «substance active employée pour prévenir ou traiter une affection ou une manifestation morbide.»

- L'utilisation du terme «médicament » dans l'information des personnes pour les recherches biomédicales doit être sans ambiguïté.

- Le produit à l'étude ne doit pas être, sauf cas particuliers, présenté comme un médicament. Par définition, le produit est en cours d'évaluation.

- La notion de «médicament expérimental» doit être précisée et explicitée, si elle est utilisée dans le document d'information. On précise que la notion de «médicament expérimental » figure dans le Code de santé publique qui en donne une définition plus « technique » que «profane».

Termes proscrits Substance pharmacologique / produit pharmacologique

\begin{tabular}{ll}
\hline 31 / PARTICIPATION \\
\hline DÉFINITION & $\begin{array}{l}\text { Fait de participer, de prendre part à la recherche bio- } \\
\text { médicale proposée. }\end{array}$ \\
\hline Exemples & $\begin{array}{l}\text { "Votre participation à cette étude est entièrement } \\
\text { libre... } \\
\text { "Votre médecin vous propose de participer à une re- } \\
\text { cherche biomédicale... . }\end{array}$ \\
\hline
\end{tabular}

Commentaires

- Notion sans difficulté. Les rédactions posent peu de problèmes et sont assez homogènes. C'est la modalité «d'être sujet de recherche » retenue par l'ensemble des rédacteurs.

Termes proscrits Coopérer/prendre part

\begin{tabular}{ll}
\hline 32 / PATIENT & \\
\hline DÉFINITION & Personne qui est l'objet de traitements ou de soins. \\
\hline Exemples & $\begin{array}{l}\text { "La modification des habitudes de vie des patients } \\
\text { ne suffit pas à diminuer ces risques... » }\end{array}$ \\
\hline
\end{tabular}

- Le terme «patient» désigne une personne considérée sous l'angle des soins. La sollicitation d'une personne pour participer à une recherche clinique est le plus souvent motivée par cette qualité. Cependant, en tant que personne participant à une recherche biomédicale, le «patient » est aussi sujet de recherche, participant ou volontaire.

- On distingue ainsi pour une même personne physique, la qualité de «personne soignée » (le patient) de la qualité de personne participante (le volontaire).

Termes proscrits Malade/ personne alitée

\begin{tabular}{|c|c|}
\hline \multicolumn{2}{|c|}{$\begin{array}{l}33 \text { / PERSONNE / sujet [de recherche] / volontaire / participant / } \\
\text { PARTICIPANT [À LA RECHERCHE] }\end{array}$} \\
\hline DÉFINITION & $\begin{array}{l}\text { Homme ou femme ayant accepté de participer à une } \\
\text { recherche biomédicale et y ayant été inclus. }\end{array}$ \\
\hline Exemples & $\begin{array}{l}\text { "Après tirage au sort, les volontaires entrant dans } \\
\text { l'étude... } \\
\text { "L'étude concerne au total } 1700 \text { personnes... } \\
\text { "L'étude peut être interrompue pour tous les partici- } \\
\text { pants... } \\
\text { «... } 36,2 \% \text { des sujets étaient abstinents...»" }\end{array}$ \\
\hline
\end{tabular}

- Le recours au terme «patient» pour désigner les personnes qui participent à des essais cliniques, par opposition à l'expression « volontaire sain » (pour les phases I) est encore fréquent.

Commentaires - Or, cette distinction n'a plus de fondement : d'une part la Loi ne désigne jamais les personnes qui se prêtent par le terme de «patient », d'autre part la distinction des recherches entre « avec bénéfice individuel direct » et «sans bénéfice individuel direct » a été abandonnée par le législateur.

- On notera aussi la tendance dans les réflexions et travaux terminologiques (Inserm par exemple) pour abandonner le terme « patient» au profit de « sujet».

- Aussi on propose de désigner, selon le contexte, la personne qui se prête à une recherche biomédicale par les seuls termes de «personne», «sujet », « participant » ou « volontaire ».

- On pourra garder, pour les phases 1 (hors cancérologie) l'expression de «volontaire sain». Dans le langage courant, on emploie fréquemment l'expression «sujet volontaire » pour désigner les sujets volontaires sains. Cet usage est incorrect puisque les malades tout aussi bien doivent être volontaires pour participer à une recherche biomédicale.

- Le terme «patients» peut-être employé quand les personnes sont décrites ou considérées en tant que telles. Parce qu'il est un marqueur fort de la situation de soin, ce terme doit être employé avec précaution.

Termes proscrits Patient / personne-sujet / sujet sain / malade / patientsujet

\section{4 / PERSONNE DE CONFIANCE}

«Toute personne majeure peut désigner une personne

DÉFINITION de confiance qui peut être un parent, un proche ou le médecin traitant, et qui sera consultée au cas où elle-même serait hors d'état d'exprimer sa volonté et de recevoir l'information nécessaire à cette fin. Cette désignation est faite par écrit. Elle est révocable à tout moment. Si le malade le souhaite, la personne de confiance l'accompagne dans ses démarches et assiste aux entretiens médicaux afin de l'aider dans ses décisions » (article L1111-6 du Code de la Santé publique). 
Exemples "En cas d'impossibilité de consentir, la personne de confiance de votre choix...»

- L'expression «personne de confiance » n'est ac-

Commentaires tuellement pas, - ou très rarement, employée en recherche biomédicale. Elle concerne cependant l'ensemble des situations où le consentement est donné par un tiers.

- On distingue ainsi la personne de confiance (qui peut intervenir dans les choix), le témoin (qui atteste que l'information a été donnée), et le représentant légal (cas particuliers). Le terme de « tiers », trop vague, est à éviter.

Termes proscrits Représentant / mandataire / répondant / décideur remplaçant

\begin{tabular}{ll}
\hline 35 / PlaCEBO & \\
\hline DÉFINITION & $\begin{array}{l}\text { Substance utilisée dans une recherche biomédicale } \\
\text { pour mesurer ou contrôler certains effets. }\end{array}$ \\
\hline Exemples & $\begin{array}{l}\text { "La composition des comprimés (X ou placebo)... » } \\
\text { "En revanche, ce bénéfice n'existe pas si vous rece- } \\
\text { vez le comprimé placebo. » }\end{array}$ \\
\hline Commentaires & $\begin{array}{l}\text { - Notion devenue familière au grand public, et } \\
\text { dont les définitions sont souvent plus compli- } \\
\text { quées que le terme lui-même. }\end{array}$
\end{tabular}

Note : le Conseil supérieur de la langue française recommande d'écrire «placébo » plutôt que «placebo ».

Termes proscrits Sans activité pharmacologique / pilule inactive / substance inactive / substance biologiquement inactive / médicament dénué d'activité pharmacologique

\section{6 / Produit Étudié / / < Nom du produit > Produit à l'étude \\ DÉFINITION Substance évaluée ou testée par la recherche biomé- dicale.

Exemples "Forme pharmaceutique qui ressemble au produit étudié.» \\ «Le <Nom du produit> a été administré à plus de cinq cents volontaires. »}

- L'habitude a été prise de désigner le produit à l'étude par le terme «traitement», qui est un marqueur de la situation de soins. Par hypothèse, le produit étudié peut se révéler inefficace et ne pas être dans ce cas un traitement.

- Aussi, chaque fois que cela est possible, on utilisera le nom du produit étudié.

- Les termes «médicament» ou «traitement» sont proscrits, sauf s'il s'agit de produits qui disposent du statut de «médicament» ou de «traitement » [voir sur ce point Médicament, Médicament expérimental, Traitement].

Termes proscrits Médicament à l'étude / traitement à l'essai / produit actif / traitement expérimental / traitement à l'étude / produit d'investigation / médicament de l'essai / nouveau traitement

\section{7 / Promoteur}

Selon la Loi du 20 décembre 1988 (Loi Huriet-

DÉFINITION Sérusclat) révisée le 9 août 2004, le promoteur est «la personne physique ou morale qui prend l'initiative d'une recherche biomédicale sur l'être humain, qui en assure la gestion et vérifie que le financement de la recherche est prévu. »

Exemples
Aventis. $~$

- Il convient d'éviter la multiplication des entités juridiques qui n'ont pas de pertinence pour l'information des personnes. Typiquement, des distinctions comme «étude organisée par XX, représenté par $\mathrm{YY} »$ sont à proscrire, ou à réserver à l'attestation de consentement.

- On propose de retenir, pour la partie «information », la dénomination «sanofi-aventis », qui accompagne le logo, avec laquelle le public est familiarisé, sauf exigences réglementaires incontournables.

Termes proscrits Sponsor (sponsorisé) / entités / représentants / personnel autorisé / personnes mandatées par lui / la société /

\section{8 / Protection des Personnes \\ DÉFINITION Ensemble des procédures et des moyens mis en œuvre pour assurer l'intégrité de la personne qui par- ticipe à une recherche biomédicale. \\ Exemples "Les comités de protection des personnes..." \\ «La Loi de protection des personnes qui se prêtent à des recherches biomédicales. »}

Commentaires

- La notion de protection des personnes est une notion juridique et législative. Elle est peu employée dans la langue profane, qui utilise davantage le concept de «sécurité ». Elle renvoie par ailleurs à un dispositif que le public ne connaît guère.

Termes proscrits Sécurité

\begin{tabular}{ll}
\hline 39 / Protocole / Protocole de l'étude \\
\hline DÉFINITION & $\begin{array}{l}\text { Description précise des conditions et du déroulement } \\
\text { de la recherche biomédicale. }\end{array}$ \\
\hline Exemples & $\begin{array}{l}\text { On évitera l'usage du terme "protocole » dans la } \\
\text { communication écrite et orale avec les personnes sol- } \\
\text { licitées de participer à la recherche. S'il est employé, } \\
\text { il doit être précisé. }\end{array}$ \\
\hline Commentaires & $\begin{array}{l}\text { Terme générique pour le soin et pour la re- } \\
\text { cherche. Le public connaît mieux les protocoles } \\
\text { de soins que les protocoles de recherche mais le } \\
\text { transfert de sens est aisé. }\end{array}$ \\
\hline
\end{tabular}

Termes proscrits Procédures de l'étude / schéma thérapeutique / schémas de traitement / schéma d'administration / brochure investigateur / protocole d'essai 


\begin{tabular}{ll}
\hline 40/ RECHERCHE BIOMÉDICALE / Étude $<+$ acronyme $>$ \\
\hline DÉFINITION & $\begin{array}{l}\text { Recherche organisée et pratiquée sur l'être humain } \\
\text { en vue du développement des connaissances biolo- } \\
\text { giques et médicales. }\end{array}$ \\
\hline$\underline{\text { Exemples }}$ & $\begin{array}{l}\text { "Pour participer à une recherche biomédicale, ... } \\
\text { "L'étude XX peut être interrompue pour tous les par- } \\
\text { ticipants par... » }\end{array}$ \\
\hline
\end{tabular}

- L'expression « recherche biomédicale » désigne à la fois un champ de pratiques (l'ensemble de Commentaires l'activité : la recherche biomédicale) et un objet singulier (une recherche biomédicale).

- On recommande de ne pas utiliser l'expression «essai thérapeutique» dans le document d'information car ce terme présente l'inconvénient de ramener l'essai dans le champ du soin.

- On évite également l'expression «essai clinique », qui appartient au langage spécialisé.

- En revanche, l'expression «étude <acronyme> » peut être employée sans difficulté tout au long du document.

Essai / essai clinique / essai de recherche clinique / Termes proscrits étude / étude clinique / expérimentation / recherche / recherche expérimentale / essai sur l'homme / projet de recherche / essai thérapeutique / protocole de recherche / étude de recherche clinique (autres : étude ancillaire / étude préliminaire)

\begin{tabular}{ll}
\hline $\mathbf{4 1} /$ RÉSULTATS DE L'ÉTUDE / résultats globaux \\
\hline DÉFINITION & $\begin{array}{l}\text { Ensemble des informations obtenues par la réalisa- } \\
\text { tion de l'étude. }\end{array}$ \\
\hline Exemples & $\begin{array}{l}\text { "Les résultats globaux de l'étude sont communi- } \\
\text { qués. » }\end{array}$ \\
\hline
\end{tabular}

- Terme qui peut renvoyer à deux sous notions :

- Les résultats personnels pour la personne qui participe ;

Commentaires

- Les résultats globaux qui portent sur l'hypothèse de départ.

- L'information pertinente pour les personnes est double :

- Quelles sont les informations personnelles susceptibles de pouvoir être communiquées au médecin traitant (nature du produit reçu, résultats d'examens, etc.);

- Quelle sera l'information reçue, et dans quelles conditions, sur l'étude elle-même.

L'évocation des résultats globaux prouve la considération accordée à la personne qui participe.

Termes proscrits Résultats personnels

\begin{tabular}{ll}
\hline 42 / RISQUES & \\
\hline Termes proscrits & $\begin{array}{l}\text { Inconvénient / effet secondaire / risques potentiels / } \\
\text { risques prévisibles / gênes / désagrément / préjudice } \\
\text { / risque médical / toxicités / événement indésirable / } \\
\text { risques inconnus / réaction indésirable }\end{array}$ \\
\hline DÉFINITION & $\begin{array}{l}\text { Ensemble des dangers et des inconvénients, plus ou } \\
\text { moins prévisibles, auxquels est exposée la personne } \\
\text { qui participe à l'étude. }\end{array}$ \\
\hline
\end{tabular}

- Notion qui pose peu de difficultés; elle renvoie aux notions voisines de « danger», «inconvénient» et «préjudice».

- L'évaluation du risque est rendue difficile par la double dimension statistique d'une part (risque alpha), et par le mode de mesure (balance bénéfice/risque).

- On propose de distinguer, dans l'information, les risques selon leur «origine»: risques dus aux produits (placebo compris), risques dus aux procédures, risques liés aux situations particulières (grossesse, etc.).

Exemples 《Les risques liés aux examens sont minimes. »

\section{3 / SECRET PROFESSIONNEL}

Termes proscrits Non documenté

DÉFINITION Obligation de ne pas divulguer des faits confidentiels appris lors du déroulement d'une recherche biomédicale, hors des cas prévus par la Loi.

- Notion familière pour le public. La règle du secret professionnel (article R5120 du Code de la Santé Publique) en vigueur dans le champ médical s'applique à celui de la recherche biomédicale.

- Cette règle s'applique à toute personne qui participe directement (médecin investigateur) ou indirectement $(A R C$, moniteurs, etc.) à une recherche biomédicale.

\begin{tabular}{l}
\hline $\begin{array}{l}\text { Exemples } \\
\text { recherche biomé du secret professionnel s'appliquent à la }\end{array}$ \\
\hline
\end{tabular}

\section{4 / SÉLECTION / visite de sélection}

Termes proscrits Apte / critères d'éligibilité / éligibilité / présélection / recrutement / période de sélection / vérifier les critères de sélection / critères d'inclusion et de non inclusion / période d'inclusion / processus de sélection / patient incluable

$\overline{\text { DÉFINITION Étape au cours de laquelle le médecin-investigateur }}$ vérifie l'éligibilité d'une personne sollicitée. 
- La procédure d'inclusion d'une personne sollicitée est mal connue du grand public. La difficulté est de parvenir à une présentation cohérente avec le vécu des personnes.

- Toute personne sollicitée n'est pas nécessairement incluse. Les différentes étapes de l'inclusion doivent être parfaitement décrites. On commande de distinguer entre visite de sélection et visite d'inclusion quand cela est possible.

Exemples «Lors de la visite de sélection, vous aurez...»

\section{5 / Signature}

Termes proscrits Signer un consentement / être invité à signer un formulaire / confirmation de l'accord de participation

DÉFINITION Élément qui atteste le consentement de la personne à sa participation à la recherche.

- Dans le dispositif actuel de protection des personnes, la signature matérialise l'accord de la personne pour participer à la recherche.

- Même après avoir signé, la personne peut cesser de consentir à tout moment, sans préjudice.

Exemples "La signature de l'attestation de consentement ne vous engage pas, ..."

«Si vous acceptez de participer, vous devez signer une attestation de consentement...»

\section{6 / SoINS}

Termes proscrits Non documenté

DÉFINITION Ensemble des actions et traitements par lesquels on conserve ou on rétablit la santé.

- Notion courante et qui pose peu de difficultés.

Commentaires

- On doit distinguer particulièrement entre la logique de soins (ordre médical) et la logique expérimentale (ordre de la recherche), qui fonde le dispositif de protection des personnes institué par la Loi.

Exemples "Si vous décidez de ne pas participer à cette étude, vous continuerez de recevoir les soins et les traitements dont vous avez besoin. »

\section{7 / SolLICITATION / proposition}

Termes proscrits Non documenté

DÉFINITION Acte de proposer à une personne de participer à une recherche biomédicale.

Commentaires

- Le terme «sollicitation » est rarement employé dans les documents d'information des personnes. Il est en revanche largement utilisé par l'ensemble des acteurs «professionnels» ou institutionnels. Le terme fréquemment utilisé pour évoquer la sollicitation est «proposition » ou « proposer».

\begin{tabular}{l}
\hline Exemples $\begin{array}{l}\text { "Le faible taux de sollicitation des personnes ralentit } \\
\text { la mise en cuvre des essais cliniques... } \\
\text { "Votre médecin vous propose de participer... . }\end{array}$ \\
\hline
\end{tabular}

\section{8 / TÉMOIN}

\begin{tabular}{ll}
\hline DÉFINITION & $\begin{array}{l}\text { Personne indépendante du promoteur et du médecin- } \\
\text { investigateur qui assiste à la présentation de l'étude à } \\
\text { la personne sollicitée, et qui atteste que la personne } \\
\text { sollicitée a bien été informée. }\end{array}$ \\
\hline Exemples & $\begin{array}{l}\text { [Formule fréquente dans les attestations de consen- } \\
\text { tement] } \\
\text { "Je soussigné, ..., en qualité de témoin, ....» }\end{array}$ \\
\hline
\end{tabular}

Commentaires

- Notion commune et juridique à la fois. Il faut distinguer entre la personne de confiance, qui porte la volonté de la personne sollicitée (désignée par elle) et le témoin, - toute personne qui pourrait, dans le principe, certifier que la procédure d'information et de recueil du consentement a eu lieu, mais qui n'a aucune légitimité pour « décider » ou « autoriser à la place de ».

- L'expression est utilisée dans l'attestation de consentement.

Termes proscrits Tierce personne

\section{9 / TIRAGE AU SORT}

DÉFINITION Procédure employée pour certaines recherches par laquelle on attribue les produits et/ou les placebos.

Exemples "Après tirage au sort, les personnes entrant dans
l'étude sont réparties en deux groupes. »

Commentaires

- L'expression «tirage au sort » est préférable à celle, plus technique de «randomisation », qui commande d'être expliquée, le plus souvent par «tirage au sort».

Termes proscrits

Aléatoire / allocation / attribution / désignation / hasard / randomisation / de façon aléatoire / choisi au hasard / affecté à / ordre aléatoire / pile ou face / essai randomisé / distribution au hasard / attribution aléatoire / répartition aléatoire / randomisation centralisée / décentralisée 


\section{0 / TRAITEMENT \\ DÉFINITION Ensemble des soins médicaux destinés à combattre la maladie pour en limiter les conséquences, éviter la mort, rétablir la santé et entraîner la guérison. \\ Exemples "Cette dernière consultation assure le retour aux soins et aux traitements habituels. \\ «Votre médecin continuera de vous prescrire les mé- dicaments et les traitements dont vous avez besoin. "}

- Notion complexe, qui regroupe les sous notions suivantes :

- Traitement standard

Commentaires

- Traitement de référence ;

- Traitement expérimental ;

Traitement curatif ;

- Traitement palliatif.
- On retrouve les ambiguïtés du terme «médicament »; on désigne en effet à la fois les produits ou substances administrées dans le cadre du soin et les produits à l'étude ( traitement à l'étude » versus «traitement courant»).

- On propose de réserver le terme «traitement» à des produits ou procédures dûment évalués. Un produit à l'étude non encore évalué ne doit pas être présenté comme un traitement dans le cadre d'une recherche expérimentale (par hypothèse).

Termes proscrits Habitudes de traitement / approches thérapeutiques / traitement à l'étude / traitement standard / traitement en première ligne / traitement classique / pratique habituelle / pratique standard / traitement de choix / traitement courant / traitement de référence / traitement curatif / traitement palliatif / traitement expérimental 